\title{
High Cyclophilin D Content of Synaptic Mitochondria Results in Increased Vulnerability to Permeability Transition
}

\author{
Kranthi Kumari Naga, ${ }^{1}$ Patrick G. Sullivan, ${ }^{1,2}$ and James W. Geddes ${ }^{1,2}$ \\ ${ }^{1}$ Spinal Cord and Brain Injury Research Center and ${ }^{2}$ Department of Anatomy and Neurobiology, University of Kentucky, Lexington, Kentucky 40536
}

\begin{abstract}
Mitochondria isolated from synaptosomes are more sensitive to $\mathrm{Ca}^{2+}$ overload and the resultant opening of the mitochondrial permeability transition pore (mPTP) than nonsynaptic mitochondria. To identify the mechanisms underlying these differences in $\mathrm{Ca}^{2+} \mathrm{dy}^{-}$ namics, we examined relative levels of mPTP components in synaptic versus nonsynaptic mitochondria. Synaptic mitochondria had higher levels of cyclophilin D when compared with nonsynaptic mitochondria, whereas levels of the voltage-dependent anion channel and the adenine nucleotide translocase were similar in the two mitochondrial fractions. These differences in $\mathrm{Ca}^{2+}$ handling between synaptic and nonsynaptic mitochondria were greatly reduced in cyclophilin D null [Ppif ${ }^{-1}$ (peptidylprolyl isomerase F)] mice. Higher concentrations of cyclosporine $\mathrm{A}$, which interacts with cyclophilin D to delay $\mathrm{mPTP}$ opening, were necessary to increase the $\mathrm{Ca}^{2+}$ uptake capacity of synaptic versus nonsynaptic mitochondria. To determine whether the differences in $\mathrm{Ca}^{2+}$ handling might reflect the relative abundance of neuronal and glial mitochondria in the two mitochondrial fractions, we compared cyclophilin D levels in primary cortical neurons and astrocytes. Primary rat cortical neurons possess higher cyclophilin D levels than do primary astrocytes. In the adult rat brain, cyclophilin D immunoreactivity was abundant in neurons but sparse in astrocytes. Together, these results demonstrate that the $\mathrm{Ca}^{2+}$ handling differences observed in synaptic versus nonsynaptic mitochondria are primarily the result of the high levels of cyclophilin $\mathrm{D}$ in synaptic mitochondria, reflecting the greater proportion of neuronal mitochondria in this fraction. The high levels of cyclophilin D in neuronal mitochondria result in their greater vulnerability to $\mathrm{MPT}$ and in higher levels of cyclosporine A being required to inhibit mPTP opening.
\end{abstract}

Key words: calcium; necrosis; cyclosporine A; Ppif; caspase-independent; astrocytes

\section{Introduction}

Mitochondria help to shape intracellular $\mathrm{Ca}^{2+}$ signaling by taking up cytosolic $\mathrm{Ca}^{2+}$ through a uniporter located on the inner mitochondrial membrane. However, their uptake capacity is finite, and, when exceeded, mitochondrial $\mathrm{Ca}^{2+}$ efflux occurs rapidly via a change in the permeability of the mitochondrial inner membrane resulting from the opening of a nonspecific channel permeable to molecules up to $1500 \mathrm{Da}$, referred to as the mitochondrial permeability transition pore (mPTP) (Haworth and Hunter, 1979; Szabo and Zoratti, 1992). Pore opening is promoted by reactive oxygen species, alkaline $\mathrm{pH}$, and inorganic phosphate (Bernardi et al., 1998). After mPTP opening, $\mathrm{Ca}^{2+}$ and other solutes are released from the matrix, the mitochondria depolarize, the matrix swells, the outer membrane ruptures, and death-effector proteins are released from the intermembrane

Received Feb. 13, 2007; revised May 1, 2007; accepted May 29, 2007.

The funding for this research was provided by National Institutes of Health Grants AG10836, NS045726, and NS058484, the Kentucky Spinal Cord and Brain Injury Research Trust, and an award from the American Heart Association. We thank Dr. Molkentin for providing breeding pairs for the Ppif ${ }^{-1-}$ mice, which were obtained through the Cincinnati Children's Research Foundation. We also thank Dr. Maile Brown for sharing her expertise in mitochondrial isolation and analysis.

Correspondence should be addressed to Dr. James W. Geddes, B379 Biomedical \& Biological Sciences Research Building, Spinal Cord and Brain Injury Research Center, 741 South Limestone Street, University of Kentucky, Lexington, KY 40536-0509. E-mail: jgeddes@uky.edu.

DOI:10.1523/JNEUROSCI.0646-07.2007

Copyright $\odot 2007$ Society for Neuroscience $\quad$ 0270-6474/07/277466-07\$15.00/0 space, leading to cell death (Kim et al., 2003; Sullivan et al., 2005; Bernardi et al., 2006).

The MPTP is thought to be composed of the outer membrane voltage-dependent anion channel (VDAC), the inner membrane adenine nucleotide translocase (ANT), and regulated by the matrix protein cyclophilin $\mathrm{D}(\mathrm{CypD})$, although additional or alternate proteins may also be involved (Crompton et al., 1998; Woodfield et al., 1998; Kokoszka et al., 2004; Bernardi et al., 2006; Baines et al., 2007). CypD, a peptidylprolyl cis-trans isomerase located within the mitochondrial matrix, facilitates a conformational change in the ANT, converting it to an "open" pore. In mice lacking the Ppif (peptidylprolyl isomerase F) gene that encodes CypD, the cells are primarily protected from necrotic, caspase-independent cell death but not from caspase-dependent apoptosis (Scorrano et al., 2002; Kim et al., 2003; Baines et al., 2005; Basso et al., 2005; Nakagawa et al., 2005).

We recently observed that mitochondria enriched from synaptosomes, isolated from rat cerebral cortex, had a lower threshold for mPTP in response to elevated $\mathrm{Ca}^{2+}$ than did the nonsynaptic pool of mitochondria (Brown et al., 2006). To investigate the mechanisms underlying the differences in $\mathrm{Ca}^{2+}$ handling in synaptic versus nonsynaptic mitochondria, we examined levels of various components of the $\mathrm{mPT}$ in synaptic and nonsynaptic mitochondria and in rat primary neurons and astrocytes. We also compared $\mathrm{Ca}^{2+}$ handling in synaptic and nonsynaptic mitochondrial populations from CypD null $\left(\mathrm{Ppif}^{-1}\right)$ mice and in 
response to cyclosporine $\mathrm{A}$ (CsA), which binds to CypD to inhibit mPTP opening (Halestrap and Davidson, 1990). We found that the CypD content of synaptic mitochondria is much greater than that of nonsynaptic mitochondria and that this difference was reduced in the absence of CypD or in the presence of high levels of CsA. In addition, we found that neuronal mitochondria contain higher levels of CypD than astrocytic mitochondria. Together, these results suggest that the greater CypD content of synaptic brain mitochondria reflects a greater proportion of neuronal mitochondria compared with the nonsynaptic mitochondrial fraction. In neuronal and synaptic mitochondria, the relatively high levels of CypD reduce the $\mathrm{Ca}^{2+}$ threshold for $\mathrm{mPT}$ and require greater CsA concentrations to inhibit mPT.

\section{Materials and Methods}

Reagents. Mannitol, sucrose, bovine serum albumin (BSA), EGTA, HEPES potassium salt, $\mathrm{KH}_{2} \mathrm{PO}_{4}, \mathrm{MgCl}_{2}$, malate, pyruvate, ADP, oligomycin A, phenylmethylsulfonyl fluoride (PMSF), carbonyl cyanide 3-chlorophenylhydrazone, and calcium chloride were purchased from Sigma (St. Louis, MO). Bicinchoninic acid (BCA) protein assay kit was purchased from Pierce (Rockford, IL). Percoll was purchased from GE Healthcare (Little Chalfont, UK). IRDye 800CW-conjugated affinitypurified anti-mouse IgG and IRDye $800 \mathrm{CW}$ and $680 \mathrm{CW}$ anti-rabbit IgG secondary antibodies were purchased from Rockland Immunochemicals (Glibertsville, PA). Calcium Green-5N hexapotassium salt (CaG5N), Hoechst 33258, and Alexa fluor secondary antibodies were purchased from Invitrogen (Carlsbad, CA), minimum essential medium, neurobasal medium, L-glutamine, B27 supplement, gentamycin, antibioticantimycotic mix, and sodium pyruvate were purchased from Invitrogen. Vectashield mounting medium was from Vector Laboratories (Burlingame, CA).

Mitochondrial isolation. All experimental protocols involving animals were approved by the University of Kentucky Animal Use and Care Committee. Mitochondrial isolation from synaptic and nonsynaptic fractions was performed using methods described previously (Brown et al., 2004, 2006). Male Sprague Dawley rats, C57BL/6 mice, and Ppif null mice (Baines et al., 2005), 3 months old, were used in these studies. After carbon dioxide asphyxiation, the rodents were decapitated, and the brains were rapidly removed. The cortices were dissected out and placed in five times the volume of ice-cold isolation buffer ( $215 \mathrm{~mm}$ mannitol, 75 mm sucrose, 0.1\% BSA, 20 mM HEPES, and 1 mm EGTA, pH adjusted to 7.2 with $\mathrm{KOH}$ ). The tissue was homogenized with a Dounce homogenizer, and an equal volume of $30 \%$ Percoll in isolation buffer was added. The resultant homogenate was layered on a discontinuous gradient of 24 and $40 \%$ Percoll and centrifuged in a Sorvall (Asheville, NC) RC-5C plus centrifuge in a fixed-angle SE-12 rotor at $30,400 \times g$ for $10 \mathrm{~min}$.

After centrifugation, band 2 (containing synaptosomes) and band 3 (nonsynaptic mitochondria) (Sims, 1990) were removed from the density gradient. Additional isolation buffer was added, and the fractions were centrifuged at $16,700 \times g$ for $15 \mathrm{~min}$. The supernatant was discarded, and the loose pellet was resuspended in $1 \mathrm{ml}$ of isolation buffer. The nonsynaptic mitochondria and synaptosomes were placed separately in a nitrogen cell disruption bomb (model 4369; Parr Instrument Company, Moline, IL) for $10 \mathrm{~min}$ at 1000 psi to disrupt the synaptosomal membrane (Brown et al., 2004). After nitrogen disruption, an equal volume of $30 \%$ Percoll was added to the individual mitochondrial fractions, and discontinuous Percoll density gradient centrifugation was performed as described above. Band 3 was obtained from each of the gradients, resuspended in isolation buffer without EGTA, and centrifuged at $16,700 \times g$ for $15 \mathrm{~min}$ and then at $13,000 \times g$ and at $10,000 \times g$ for $10 \mathrm{~min}$ each (Brown et al., 2006). The final mitochondrial pellet was resuspended in isolation buffer without EGTA containing a protease inhibitor cocktail (Complete Mini; Roche Applied Science, Indianapolis, IN) and stored on ice. Protein concentration was determined using the BCA protein assay (Pierce).

Western blotting. Isolated nonsynaptic and synaptic mitochondria were resuspended in isolation buffer containing protease inhibitors with
$0.1 \%$ Triton X-100, sonicated for $20 \mathrm{~s}$, and centrifuged at $10,000 \times g$ for $10 \mathrm{~min}$. The supernatant was used for Western blots. Equal amounts of protein $(20 \mu \mathrm{g})$ were added in each lane, and the proteins were separated by SDS-PAGE using 12\% Tris-acrylamide/bis gels, along with molecular weight markers. After SDS-PAGE, polypeptides were transferred electrophoretically onto $0.2 \mu \mathrm{m}$ nitrocellulose membranes. Membranes were incubated at room temperature for $1 \mathrm{~h}$ in $5 \%$ nonfat milk in $50 \mathrm{~mm}$ Tris-saline at pH 7.5 (TBS). The membranes were incubated overnight with one mouse monoclonal primary antibody and one rabbit polyclonal antibody and diluted in 5\% nonfat milk in $50 \mathrm{~mm}$ Tris-saline containing $0.05 \%$ Tween 20 (TTBS), $\mathrm{pH} 7.5$, at $22^{\circ} \mathrm{C}$. After overnight incubation, the membranes were rinsed three times in TTBS and incubated in $680 \mathrm{CW}$ or $800 \mathrm{CW}$ anti-rabbit and/or $800 \mathrm{CW}$ anti-mouse IgG (H\&L) secondary antibodies in $5 \%$ nonfat milk in TTBS, $\mathrm{pH} 7.5$, at $22^{\circ} \mathrm{C}$. The blots were visualized using a LI-COR Biosciences (Lincoln, NE) Odyssey infrared imaging system. After visualization, the blots were stripped by rinsing in TBS and incubated at $55^{\circ} \mathrm{C}$ for $30 \mathrm{~min}$. in a buffer containing $200 \mathrm{~mm}$ glycine, $2 \%$ SDS, and $100 \mathrm{~mm} \beta$-mercaptoethanol. Membranes were rinsed $2 \times$ in TBS and then blocked and reprobed with an additional primary antibody. The primary antibodies were directed against the following: VDAC (rabbit polyclonal; Affinity BioReagents, Golden, CO), cytochrome oxidase subunit IV (COX IV; mouse monoclonal; Invitrogen), ANT (mouse monoclonal; Santa Cruz Biotechnology, Santa Cruz, CA), CypD (mouse monoclonal; EMD Biosciences/Calbiochem, San Diego, CA), glial fibrillary acidic protein (GFAP) (rabbit polyclonal; Affinity BioReagents), neuronal-specific nuclear protein (NeuN) (mouse monoclonal; Millipore/Chemicon, Temecula, CA), and mitochondrial heat shock protein (mHSP70) (mouse monoclonal; Affinity BioReagents). For mitochondrial fractions, the initial incubation typically contained antibodies against VDAC (rabbit) and CypD (mouse), with mHSP70 examined after stripping and reprobing. Other membranes were first probed for ANT and then for CypD. For neuronal and astrocyte lysates, membranes were first probed for GFAP (rabbit) and CypD (mouse) and then for COX IV (mouse) or for CypD and then NeuN.

Spectrofluorophotometric assays. Spectrofluorophotometric assays were performed as described previously (Brown et al., 2006). Briefly, nonsynaptic and synaptic mitochondrial fractions (50 $\mu \mathrm{g}$ protein $/ \mathrm{ml})$ were placed in $\mathrm{KCl}$ respiration buffer containing $100 \mathrm{~nm} \mathrm{CaG5N}$ to monitor extramitochondrial $\mathrm{Ca}^{2+}$, in a constantly stirred, temperaturecontrolled cuvette at $37^{\circ} \mathrm{C}$. The cuvette was placed in a Shimadzu (Kyoto, Japan) RF-5301PC spectrofluorophotometer, with excitation at $506 \mathrm{~nm}$ and emission at $532 \mathrm{~nm}$. For each scan, a baseline reading was obtained after the addition of $5 \mathrm{~mm}$ pyruvate and $2.5 \mathrm{~mm}$ malate at $1 \mathrm{~min}$, then 150 $\mu \mathrm{M}$ ADP at $2 \mathrm{~min}$, and then $1 \mu \mathrm{M}$ oligomycin at $3 \mathrm{~min}$. At $5 \mathrm{~min}, \mathrm{Ca}^{2+}$ was slowly infused ( $160 \mathrm{nmol} \mathrm{Ca}^{2+} / \mathrm{mg}$ protein per minute) using a $\mathrm{KD}$ Scientific (Holliston, MA) model 310 series infusion syringe pump (Zoccarato and Nicholls, 1982; Chalmers and Nicholls, 2003; Brown et al., 2006) until the mitochondria were no longer able to sequester $\mathrm{Ca}^{2+}$, as indicated by a rapid rise in the CaG5N signal. $\mathrm{Ca}^{2+}$ uptake capacity was calculated as amount of $\mathrm{Ca}^{2+}$ infused before the point at which the CaG5N signal was $150 \%$ above the baseline reading, as in our previous study (Brown et al., 2006).

Cell culture. Primary neuronal cultures of fetal rat [embryonic day 18 (E18)] cortical neurons were prepared as described previously (Pang et al., 2003; Sengoku et al., 2004) and maintained for $7 \mathrm{~d}$ in vitro in Neurobasal with B27 (Brewer et al., 1993) at $37^{\circ} \mathrm{C}$ in a humidified incubator with $5 \% \mathrm{CO}_{2}$ and $95 \%$ air. Primary astrocyte cultures were prepared from the same cell suspension as primary neurons and were seeded onto culture flasks containing minimum essential medium with Earle's salts and L-glutamine (Invitrogen) with 10\% fetal bovine serum (HyClone, Logan, UT).

To prepare lysates for Western blotting, the cells were washed once in ice-cold PBS with $1 \mathrm{mM}$ PMSF. The cells were extracted in lysis buffer (62.5 mm Tris, $6 \mathrm{~m}$ urea, 10\% glycerol, and 2\% SDS) using a cell scraper and sonicated for $10 \mathrm{~s}$ at $10 \%$ amplitude (Branson 250 Digital Sonifier, model 102C converter; Branson Ultrasonics, Danbury, CT). The lysates were centrifuged at $4^{\circ} \mathrm{C}$ for $10 \mathrm{~min}, 2000 \mathrm{rpm}$ (Eppendorf $5415 \mathrm{D}$ centrifuge), and the supernatants were used for Western blots. Protein content was determined using the BCA assay (Pierce). 


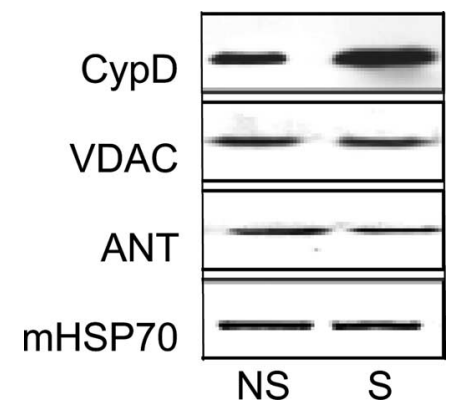

Figure 1. Synaptic mitochondria contain a higher content of cyclophilin D than nonsynaptic mitochondria. Synaptic (S) and nonsynaptic (NS) mitochondrial fractions probed by Western blot for CypD and additional mitochondrial proteins, including VDAC, ANT, and mHSP70. Levels of cyclophilin D were significantly higher in synaptic when compared with nonsynaptic mitochondrial fractions, whereas levels of other mitochondrial proteins examined were similar in the two fractions. Quantitative data are presented in Results.

Immunohistochemistry. Male Sprague Dawley rats were anesthetized with pentobarbital and perfused transcardially with PBS followed by $4 \%$ paraformaldehyde in $0.1 \mathrm{M}$ phosphate buffer, $\mathrm{pH}$ 7.4. Brains were removed and immersed overnight in the same fixative at $4^{\circ} \mathrm{C}$ and subsequently cryoprotected at $4^{\circ} \mathrm{C}$ in PBS containing $30 \%$ sucrose. Coronal brain slices, $40 \mu \mathrm{m}$, were prepared using a freezing sliding microtome (MicromHM 400 R; Zeiss, Thornwood, NY). Free-floating sections were incubated in PBS containing 0.2\% Triton X-100 followed by incubation in PBS containing either goat or horse serum, corresponding to the source of the secondary antibodies used, for $30 \mathrm{~min}$. Sections were rinsed in PBS and incubated overnight in PBS with primary antibody and blocking serum. The primary antibodies included anti-CypD (Calbiochem) and anti-GFAP (Affinity BioReagents). Sections were then rinsed two times in PBS and incubated with biotinylated anti-mouse IgG (Vector Laboratories) and Alexa fluor 594 anti-rabbit secondary antibodies (Invitrogen) for $60 \mathrm{~min}$. After incubation in the secondary antibodies, the sections were rinsed three times in PBS and incubated in streptavidin Alexa fluor 488 conjugate for $30 \mathrm{~min}$, followed by rinsing two times in $\mathrm{dH}_{2} \mathrm{O}$ and incubation in Hoechst 33258 (Invitrogen) in PBS for 15 min to stain nuclei. After rinsing three times in $\mathrm{dH}_{2} \mathrm{O}$, the free-floating sections were collected on microscope slides, coverslipped using Vectashield mounting medium (Vector Laboratories), and imaged using a Leica TCS SP5 laser scanning confocal microscope. Controls included omission of the primary antibody, use of species inappropriate secondary antibodies, and single-label immunocytochemistry.

Statistics. Statistical analyses were performed using either an unpaired $t$ test or a one-way ANOVA and Scheffé's post hoc analysis when appropriate. Results were considered significant when $p<0.05$. The results are expressed as the group means \pm SEM from at least three independent experiments. The group size for each experiment is indicated in the figure legends.

\section{Results}

\section{Increased CypD content in synaptic mitochondria}

To investigate the mechanisms underlying the reduced $\mathrm{mPT}$ threshold in synaptic mitochondria, we examined relative levels of mPTP components and of the mitochondrial matrix protein mHSP70 in synaptic and nonsynaptic mitochondria (Fig. 1). Western blot analysis revealed that the content of CypD in the synaptic mitochondrial fraction was 2.1-fold greater than in nonsynaptic mitochondria (Fig. 1) $(p<0.001)$. In contrast, levels of VDAC, ANT, and mHSP70 were similar in the two mitochondrial populations (ratio of synaptic vs nonsynaptic was $1.061, p=$ 0.640 for VDAC; $1.36, p=0.324$ for ANT; $0.83, p=0.146$ for $\mathrm{mHSP} 70 ; n=3-5$ per group).
Synaptic mitochondria require higher CsA concentrations to inhibit $\mathrm{mPT}$

CsA binds to CypD and inhibits mPT opening by interfering with the conformational change in ANT that favors the $\mathrm{mPT}$ (Halestrap and Davidson, 1990). The greater levels of CypD in synaptic mitochondria suggest that higher CsA concentrations would be necessary to inhibit mPT compared with nonsynaptic mitochondria. In support, $1 \mu \mathrm{M}$ CsA significantly increased $\mathrm{Ca}^{2+}$ accumulation in nonsynaptic $(p=0.002)$, but not in synaptic $(p=0.522$ ), mitochondria (Fig. 2), similar to results observed previously (Brown et al., 2006). In nonsynaptic mitochondria, $\mathrm{Ca}^{2+}$ accumulation in the presence of $1 \mu \mathrm{M}$ CsA was similar to that observed with $5 \mu \mathrm{M}$ CsA (Fig. 2) ( $p=0.717)$. In contrast, 5 $\mu \mathrm{M}$ CsA significantly improved the ability of synaptic mitochondria to accumulate $\mathrm{Ca}^{2+}$ compared with results obtained with 1 $\mu \mathrm{M}$ CsA (Fig. 2) $(p<0.001)$. At $2.5 \mu \mathrm{M}$ CsA, the $\mathrm{Ca}^{2+}$ uptake capacity of synaptic mitochondria was greater than observed with $1 \mu \mathrm{M}$ but less than obtained with $5 \mu \mathrm{M}$ (results not shown). Additional increases in CsA beyond $5 \mu \mathrm{M}$ had no effect on $\mathrm{Ca}^{2+}$ buffering capacity in either synaptic or nonsynaptic mitochondria (results not shown). In the presence of $5 \mu \mathrm{M} \mathrm{CsA}$, the $\mathrm{Ca}^{2+}$ uptake capacity of nonsynaptic mitochondria was $43 \%$ greater than that of synaptic mitochondria $(p=0.001)$, much less than the 15 -fold difference in the $\mathrm{Ca}^{2+}$ uptake capacity of synaptic and nonsynaptic mitochondria observed in the absence of CsA (Fig. 2C).

\section{Synaptic and nonsynaptic $\mathrm{Ca}^{2+}$ buffering differences are attenuated in Ppif $^{-1-}$ mice}

The above results suggest that differences in the $\mathrm{Ca}^{2+}$ buffering ability of synaptic versus nonsynaptic mitochondria result from differing levels of CypD in the two mitochondrial populations. We therefore examined the $\mathrm{Ca}^{2+}$ buffering ability of synaptic versus nonsynaptic mitochondria obtained from wild-type (C57BL/6) and CypD-deficient (Ppif ${ }^{-1}$ ) mice (Baines et al., 2005). The $\mathrm{Ca}^{2+}$ uptake capacity of nonsynaptic mitochondria isolated from the cortex of C57BL/6 mice was 4.3-fold greater than that of synaptic mitochondria (Fig. 3) ( $p<0.001$ ). In contrast, the difference in $\mathrm{Ca}^{2+}$ uptake capacity of nonsynaptic versus synaptic mitochondrial fractions obtained from $P_{p i f^{-/}}$mice was reduced to 1.69 -fold (Fig. 3) ( $p=0.001)$. In both the synaptic and nonsynaptic mitochondrial fractions, $\mathrm{Ca}^{2+}$ uptake capacity was greater in the Ppif ${ }^{-1}$ mice compared with wild-type C57BL/6 mice (Fig. 3) ( $p=0.003$ for nonsynaptic mitochondria; $p=0.001$ for synaptic mitochondria).

\section{High levels of CypD in neurons, low levels in astrocytes}

The above results suggest that the reduced $\mathrm{Ca}^{2+}$ uptake capacity of synaptic mitochondria obtained from rat and wild-type mouse cortex compared with nonsynaptic mitochondria from the same sources may reflect the greater CypD content of synaptic mitochondria. Synaptic mitochondria are thought to be predominantly neuronal in origin (Brown et al., 2006). Nonsynaptic mitochondria are derived from both neurons and glia. Thus, the greater level of CypD in synaptic mitochondria may result from higher levels of CypD in neuronal versus glial mitochondria. We therefore examined the levels of CypD in primary neuron and astrocyte cultures. Western blots, using antibodies against NeuN (Mullen et al., 1992) and GFAP, confirmed that the cultures were predominantly neuronal or astrocytic (Fig. 4). CypD levels were 2.6 -fold greater $(p=0.003)$ in the primary neurons versus astrocytes (Fig. 4). In contrast, this ratio was 0.9 for $\operatorname{COX~IV~(~} p=$ 
0.301), indicating equivalent loading of mitochondrial proteins in the primary neuron and astrocyte lysates.

To determine whether these differences in the CypD content of neurons versus astrocytes exist in adult rat brain, we used immunocytochemistry to examine the distribution of CypD in the brain of 3-month-old male, Sprague Dawley rats. This evaluation focused on the hippocampus because of well defined neuronal layers in this region. Within the hippocampal formation, CypD immunoreactivity was punctate and distributed across both cellular and molecular layers (Fig. 5), similar to the distribution of cytochrome oxidase and cytochrome $c$ (Kageyama and WongRiley, 1982; Hevner and Wong-Riley, 1989; Gulyas et al., 2006). Within neuronal layers, including stratum pyramidale and the granule cell layer, CypD was present in neuronal soma and appeared as a band of immunoreactivity associated with each layer. In individual neurons, punctuate CypD immunoreactivity surrounded the nucleus. In some neurons, robust CypD immunostaining was observed (Fig. 5B). This is similar to results obtained for cytochrome $c$ immunohistochemistry, in which the intensely stained neurons were found to be parvalbumin-immunoreactive inhibitory neurons (Gulyas et al., 2006). In some neurons, CypD could be observed in neurites (Fig. $5 B$ ).

To determine the possible colocalization of CypD immunoreactivity in astrocytes, sections were double labeled with antibodies against CypD and GFAP, and cell nuclei were stained with Hoechst 33258 (Fig. 5 B, C). CypD immunoreactivity was detectable, although sparse, in the soma of GFAP-positive cells. In astrocytes, CypD was localized near the soma, consistent with the observation that narrow width of astrocytic filopodia and lamellipodia excludes mitochondria from these processes (Hertz et al., 2007).

\section{Discussion}

The results of this study demonstrate that the greater CypD content of synaptic mitochondria compared with nonsynaptic mitochondria represents a major factor underlying the decreased ability of synaptic mitochondria to sequester $\mathrm{Ca}^{2+}$ before undergoing mPT (Brown et al., 2006). The absence of CypD, or presence of $5 \mu \mathrm{M}$ CsA, greatly reduced differences in the $\mathrm{Ca}^{2+}$ uptake capacity of the two mitochondrial fractions. The results further illustrate that the greater CypD content of synaptic mitochondria likely reflects the greater proportion of neuronal mitochondria in this fraction. The high CypD content of primary rat cortical neurons, and low content in primary astrocytes, observed in the present study is consistent with the different $\mathrm{Ca}^{2+}$ uptake capacity of these cell types. Primary rat cortical astrocytes have a greater $\mathrm{Ca}^{2+}$ uptake capacity than do cerebellar granule neurons,
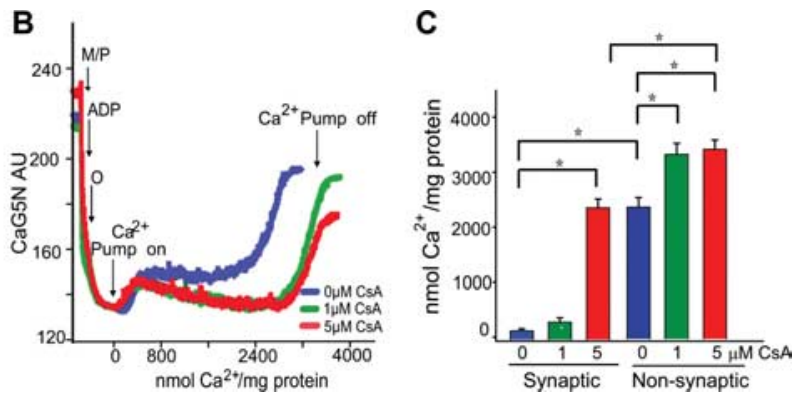

Figure 2. Greater cyclosporine A concentrations are required to increase $\mathrm{Ca}^{2+}$ uptake capacity in synaptic versus nonsynaptic mitochondrial fractions. Isolated synaptic or nonsynaptic mitochondria, in the presence or absence of 1 or $5 \mu \mathrm{m} C s \mathrm{~A}$, were placed maximal $\Delta \Psi_{\mathrm{m}}$. Ca ${ }^{2+}$ infusion began at $5 \mathrm{~min}$ (160 $\mathrm{nmol} \mathrm{Ca}{ }^{2+} / \mathrm{mg}$ protein per minute), causing a small, initial increase in

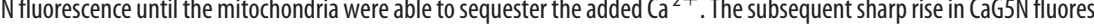
$\boldsymbol{B}$, In the absence of cyclosporine, synaptic mitochondria $(\boldsymbol{A})$ sequestered much less $\mathrm{Ca}^{2+}$ than nonsynaptic mitochondria $(\boldsymbol{B})$ ore undergoing permeability transition, as reported previously (Brown et al., 2006). AU, Arbitrary units. Arrows indicate the mitochondrial protein before permeability transition; $n=4$ per group. One micromolar cyclosporine A significantly increased the $\mathrm{Ca}^{2+}$ uptake capacity of nonsynaptic mitochondria before permeability transition but did not influence the $\mathrm{Ca}^{2+}$ uptake capacity of synaptic mitochondria. Increasing the cyclosporine A concentration to $5 \mu \mathrm{m}$ significantly increased the $\mathrm{Ca}^{2+}$ uptake capacity of synaptic mitochondria compared with both 0 and $1 \mu \mathrm{m}$ cyclosporine A. In contrast, the higher cyclosporine A concentration did not A. In the presence of $5 \mu \mathrm{m}$ cyclosporine $\mathrm{A}$, the $\mathrm{Ca}^{2+}$ uptake capacity of nonsynaptic mitochondria remained greater than that of

A

Figure 3. In cyclophilin D null $\left(\right.$ Ppif $\left.^{-1-}\right)$ mice, the $\mathrm{Ca}^{2+}$ uptake capacity of synaptic mitochondria is increased. Isolated synaptic or nonsynaptic mitochondria from wild-type $\mathrm{C}_{\mathrm{BBL}} / 6$ or Ppif ${ }^{-1}$ mice (Baines et al., 2005) were placed in a constantly stirred, temperature-controlled cuvette as in Figure 2. A, Representative traces of CaG5N fluorescence for synaptic mitochondria. B, Nonsynaptic mitochondria. AU, Arbitrary units. C, Quantitative results from five animals in each group ( $\left.{ }^{*} p<0.05\right)$. Synaptic mitochondria from Ppif ${ }^{-1}$ mice were able to sequester more $\mathrm{Ca}^{2+}$ than wild-type $\mathrm{C57BL/6}$ mice, before undergoing permeability transition indicated by the rapid rise in the CaG5N fluorescent signal. The Ca ${ }^{2+}$ uptake capacity of However, the $\mathrm{Ca}^{2+}$ uptake capacities of synaptic and nonsynaptic mitochondria were significantly different in the CypD-deficient Ppif $^{-1-}$ mice. and this uptake capacity is enhanced by CsA in astrocytes but not in neurons (Bambrick et al., 2006). Also consistent with the low cyclophilin D content of astrocytic mitochondria is their relative resistance to large cytosolic $\mathrm{Ca}^{2+}$ loads (Kahlert et al., 2001).

Previously, a role for CypD in the impaired $\mathrm{Ca}^{2+}$ handling of synaptic mitochondria had been questioned based on the inability of $1 \mu \mathrm{M}$ CsA to increase $\mathrm{Ca}^{2+}$ uptake capacity (Brown et al., 2006). The present study demonstrates the ability of synaptic mitochondria to sequester $\mathrm{Ca}^{2+}$ is improved by $5 \mu \mathrm{M} \mathrm{CsA}$ and in CypD-deficient (Ppif ${ }^{-1}$ ) mice. CsA, at concentrations at or below $100 \mathrm{nM}$, is a potent inhibitor of permeability transition in liver mitochondria (Broekemeier et al., 1989). In contrast, brain mitochondria appear resistant to $\mathrm{mPT}$ inhibition by CsA in some studies (Kristal and Dubinsky, 1997; Brustovetsky and Dubinsky, 


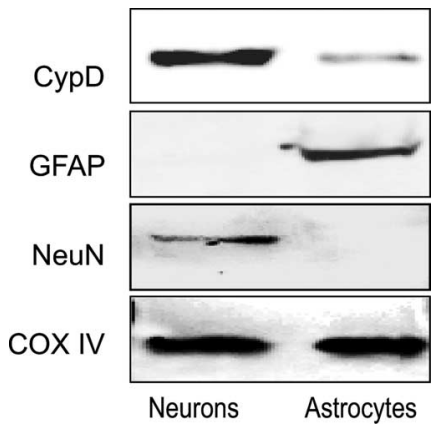

Figure 4. Primary rat cortical neurons contain higher levels of cyclophilin D than primary astrocytes. In lysates of primary rat cortical neurons and astrocyte cultures, prepared from E18 rats, $7 \mathrm{~d}$ in vitro, levels of mitochondrial proteins were evaluated by Western blot as in Figure 1 . The predominance of neurons and astrocytes in the respective primary cultures was confirmed with antibodies against NeuN and GFAP. Western blots of the nuclear encoded COX IV indicate similar mitochondrial content in the two cellular homogenates. Representative Western blots are shown in $\boldsymbol{A}$. Quantitative data are described in Results.
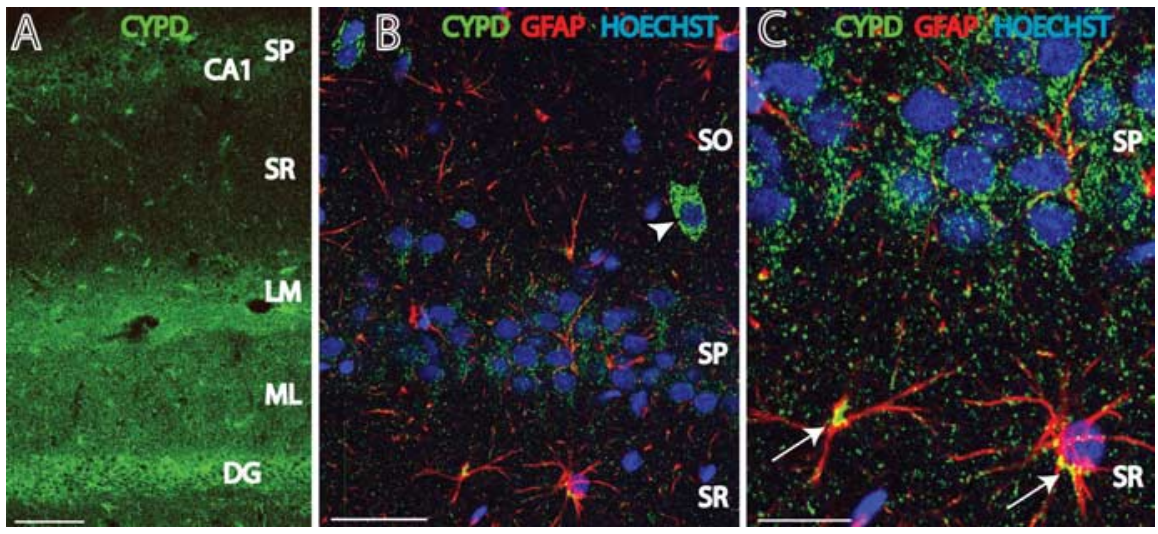

Figure 5. Cyclophilin $D$ is abundant in neurons in adult rat brain. $\boldsymbol{A}-\boldsymbol{C}$, Confocal images obtained from coronal sections in the hippocampal region of a male Sprague Dawley rats, aged 3 months. $\boldsymbol{B}$ and $\boldsymbol{C}$ are $2 \mu \mathrm{m}$ optical sections. $\boldsymbol{A}$, In the hippocampus, CypD immunoreactivity was evident in neuronal layers, including the dentate gyrus granule cell layer (DG) and CA1 stratum pyramidale (SP). Immunoreactivity was also prominent in the dentate gyrus molecular layer (ML) and stratum lacunosommoleculare of CA1 (LM), in which it was difficult to associate immunoreactivity with dendrites, axons, or astrocytes. $\boldsymbol{B}, A$ highermagnification image of $C A 1$ reveals punctuate immunoreactivity surrounding pyramidal neurons and occasional neurons (arrowhead) exhibiting robust immunoreactivity. (ell nuclei are labeled with Hoechst 33258. Within these neurons, the punctuate immunoreactivity surrounding the nucleus is consistent with the mitochondrial localization of cyclophilin D. In stratum radiatum (SR) and stratum oriens (SO), immunoreactivity was relatively sparse but was evident in astrocytes double labeled for GFAP. C, This is more clearly observed in an enlarged view, in which the arrows identify cyclophilin D immunoreactivity associated with GFAP-labeled astrocytes. However, much of the cyclophilin D immunoreactivity in stratum radiatum and stratum oriens did not colocalize with GFAP and is therefore thought to represent axonal, dendritic, or synaptic localization. Scale bars: $\boldsymbol{A}, 100 \mu \mathrm{m} ; \boldsymbol{B}, 50$ $\mu \mathrm{m} ; \mathrm{C}, 25 \mu \mathrm{m}$.

2000; Kristian et al., 2000; Chinopoulos et al., 2003) but not in others (Hansson et al., 2004a). These discrepancies may relate to the conditions used, including the presence or absence of ADP, oligomycin, and BSA in the respiring mitochondrial preparations (Brustovetsky and Dubinsky, 2000; Hansson et al., 2003). In the presence of $0.1 \% \mathrm{BSA}, 150 \mu \mathrm{M} \mathrm{ADP}$, and $1 \mu \mathrm{M}$ oligomycin, $1 \mu \mathrm{M}$ CsA inhibited $\mathrm{Ca}^{2+}$-induced $\mathrm{mPT}$ in nonsynaptosomal mitochondria, consistent with results obtained by Hansson et al. (2004a). However, under these same conditions, $1 \mu \mathrm{M}$ CsA was ineffective in synaptic mitochondria, and it was necessary to increase the CsA concentration to improve $\mathrm{Ca}^{2+}$ sequestration and inhibit mPT because of the high CypD content of synaptic mitochondria. These results suggest that the contrasting results obtained previously regarding the ability of CsA to inhibit $\mathrm{MPT}$ in isolated brain mitochondria may reflect, at least in part, the relative abundance of neuronal versus glial mitochondria in the mi- tochondrial preparations, with neuronal mitochondria being more vulnerable to $\mathrm{mPT}$ but requiring higher CsA levels to inmPTP opening.

mough the variation in CypD content in the mitochondria questions remain unanswered. It is not known whether the greater CypD content in neurons versus astrocytes reflects CypD levels in all mitochondria or in a subset of mitochondria, in the two cell types. Whether mitochondria from nerve terminals have higher CypD and impaired $\mathrm{Ca}^{2+}$ handling compared with mitochondria from neuronal somata remains unanswered. Finally, the difference in $\mathrm{Ca}^{2+}$ uptake capacity that persists in the absence of CypD or presence of $5 \mu \mathrm{M}$ CsA suggests that additional mechanisms contribute to differences in $\mathrm{Ca}^{2+}$ cycling of synaptic and nonsynaptic mitochondria.

The greater CsA concentration required to inhibit $\mathrm{mPTP}$ opening in synaptic mitochondria compared with nonsynaptic brain mitochondria and liver mitochondria has important implications with regard to the use of this compound and derivatives as neuroprotective agents. In vitro studies demonstrate that CsA is protective against excitotoxic neurodegeneration induced by glutamate, NMDA, or hypoglycemia (Nieminen et al., 1996; Schinder et al., 1996; White and Reynolds, 1996; Friberg et al., 1998). CsA also inhibits calcineurin, but the CsA-mediated inhibition of $\mathrm{mPT}$ contributes to neuroprotection, as demonstrated by CsA analogs that do not inhibit calcineurin (Friberg et al., 1998; Khaspekov et al., 1999; Fox et al., 2003; Hansson et al., 2004b). In vivo, the results are less clear, and the role of $\mathrm{mPT}$ in neuron death has been contentious (Sullivan et al., 2005). Numerous studies have examined the ability of CsA to protect against neurodegeneration after a variety of insults. However, the neuroprotection achieved is often partial and highly dependent on the CsA dose and route of administration (Brustovetsky and Dubinsky, 2000; Matsumoto et al., 2002; Okonkwo et al., 2003). CsA is only weakly permeable across the blood-brain barrier; intracarotid administration or disruption of the blood-brain barrier is necessary to achieve high brain CsA levels (Matsumoto et al., 2002; Sinigaglia-Coimbra et al., 2002; Vachon et al., 2002). Moreover, CsA is neurotoxic at high concentrations (McDonald et al., 1996; Gijtenbeek et al., 1999). Thus, using systemic administration of CsA, it is difficult to achieve brain concentrations sufficient to inhibit $\mathrm{mPT}$ in synaptic mitochondria. Non-immunosuppressive CsA analogs, which do not inhibit calcineurin and exhibit reduced neurotoxicity, appear promising (Hansson et al., 2004b; Sullivan et al., 2005).

In conclusion, the present data demonstrate that the high levels of CypD in synaptic mitochondria and neurons and low levels in astrocytes and nonsynaptic mitochondria influence both mitochondrial $\mathrm{Ca}^{2+}$ buffering capacity and levels of CsA required to inhibit mPT. Importantly, the results indicate that high CsA concentrations are necessary to protect neuronal and synaptic mitochondria against insults that induce mPT. 


\section{References}

Baines CP, Kaiser RA, Purcell NH, Blair NS, Osinska H, Hambleton MA, Brunskill EW, Sayen MR, Gottlieb RA, Dorn GW, Robbins J, Molkentin JD (2005) Loss of cyclophilin D reveals a critical role for mitochondrial permeability transition in cell death. Nature 434:658-662.

Baines CP, Kaiser RA, Sheiko T, Craigen WJ, Molkentin JD (2007) Voltagedependent anion channels are dispensable for mitochondrial-dependent cell death. Nat Cell Biol 9:550-555

Bambrick LL, Chandrasekaran K, Mehrabian Z, Wright C, Krueger BK, Fiskum G (2006) Cyclosporin A increases mitochondrial calcium uptake capacity in cortical astrocytes but not cerebellar granule neurons. J Bioenerg Biomembr 38:43-47.

Basso E, Fante L, Fowlkes J, Petronilli V, Forte MA, Bernardi P (2005) Properties of the permeability transition pore in mitochondria devoid of Cyclophilin D. J Biol Chem 280:18558-18561.

Bernardi P, Colonna R, Costantini P, Eriksson O, Fontaine E, Ichas F, Massari S, Nicolli A, Petronilli V, Scorrano L (1998) The mitochondrial permeability transition. Biofactors 8:273-281.

Bernardi P, Krauskopf A, Basso E, Petronilli V, Blachly-Dyson E, Di Lisa F, Forte MA (2006) The mitochondrial permeability transition from in vitro artifact to disease target. FEBS J 273:2077-2099.

Brewer GJ, Torricelli JR, Evege EK, Price PJ (1993) Optimized survival of hippocampal neurons in B27-supplemented Neurobasal, a new serumfree medium combination. J Neurosci Res 35:567-576.

Broekemeier KM, Dempsey ME, Pfeiffer DR (1989) Cyclosporin A is a potent inhibitor of the inner membrane permeability transition in liver mitochondria. J Biol Chem 264:7826-7830.

Brown MR, Sullivan PG, Dorenbos KA, Modafferi EA, Geddes JW, Steward O (2004) Nitrogen disruption of synaptoneurosomes: an alternative method to isolate brain mitochondria. J Neurosci Methods 137:299-303.

Brown MR, Sullivan PG, Geddes JW (2006) Synaptic mitochondria are more susceptible to $\mathrm{Ca}^{2+}$ overload than nonsynaptic mitochondria. J Biol Chem 281:11658-11668.

Brustovetsky N, Dubinsky JM (2000) Limitations of cyclosporin A inhibition of the permeability transition in CNS mitochondria. J Neurosci 20:8229-8237.

Chalmers S, Nicholls DG (2003) The relationship between free and total calcium concentrations in the matrix of liver and brain mitochondria. J Biol Chem 278:19062-19070.

Chinopoulos C, Starkov AA, Fiskum G (2003) Cyclosporin A-insensitive permeability transition in brain mitochondria: inhibition by 2-aminoethoxydiphenyl borate. J Biol Chem 278:27382-27389.

Crompton M, Virji S, Ward JM (1998) Cyclophilin-D binds strongly to complexes of the voltage-dependent anion channel and the adenine nucleotide translocase to form the permeability transition pore. Eur J Biochem 258:729-735.

Fox DA, Poblenz AT, He L, Harris JB, Medrano CJ (2003) Pharmacological strategies to block rod photoreceptor apoptosis caused by calcium overload: a mechanistic target-site approach to neuroprotection. Eur J Ophthalmol 13 [Suppl 3]:S44-S56.

Friberg H, Ferrand-Drake M, Bengtsson F, Halestrap AP, Wieloch T (1998) Cyclosporin A, but not FK 506, protects mitochondria and neurons against hypoglycemic damage and implicates the mitochondrial permeability transition in cell death. J Neurosci 18:5151-5159.

Gijtenbeek JM, van den Bent MJ, Vecht CJ (1999) Cyclosporine neurotoxicity: a review. J Neurol 246:339-346.

Gulyas AI, Buzsaki G, Freund TF, Hirase H (2006) Populations of hippocampal inhibitory neurons express different levels of cytochrome c. Eur J Neurosci 23:2581-2594.

Halestrap AP, Davidson AM (1990) Inhibition of $\mathrm{Ca}^{2+}$-induced largeamplitude swelling of liver and heart mitochondria by cyclosporin is probably caused by the inhibitor binding to mitochondrial-matrix peptidyl-prolyl cis-trans isomerase and preventing it interacting with the adenine nucleotide translocase. Biochem J 268:153-160.

Hansson MJ, Persson T, Friberg H, Keep MF, Rees A, Wieloch T, Elmer E (2003) Powerful cyclosporin inhibition of calcium-induced permeability transition in brain mitochondria. Brain Res 960:99-111.

Hansson MJ, Mansson R, Mattiasson G, Ohlsson J, Karlsson J, Keep MF, Elmer E (2004a) Brain-derived respiring mitochondria exhibit homo- geneous, complete and cyclosporin-sensitive permeability transition. J Neurochem 89:715-729.

Hansson MJ, Mattiasson G, Mansson R, Karlsson J, Keep MF, Waldmeier P, Ruegg UT, Dumont JM, Besseghir K, Elmer E (2004b) The nonimmunosuppressive cyclosporin analogs NIM811 and UNIL025 display nanomolar potencies on permeability transition in brain-derived mitochondria. J Bioenerg Biomembr 36:407-413.

Haworth RA, Hunter DR (1979) The $\mathrm{Ca}^{2+}$-induced membrane transition in mitochondria. II. Nature of the $\mathrm{Ca}^{2+}$ trigger site. Arch Biochem Biophys 195:460-467.

Hertz L, Peng L, Dienel GA (2007) Energy metabolism in astrocytes: high rate of oxidative metabolism and spatiotemporal dependence on glycolysis/glycogenolysis. J Cereb Blood Flow Metab 27:219-249.

Hevner RF, Wong-Riley MT (1989) Brain cytochrome oxidase: purification, antibody production, and immunohistochemical/histochemical correlations in the CNS. J Neurosci 9:3884-3898.

Kageyama GH, Wong-Riley MT (1982) Histochemical localization of cytochrome oxidase in the hippocampus: correlation with specific neuronal types and afferent pathways. Neuroscience 7:2337-2361.

Kahlert S, Schild L, Reiser G (2001) Mitochondrial polarization in rat hippocampal astrocytes is resistant to cytosolic $\mathrm{Ca}^{2+}$ loads. J Neurosci Res 66:1019-1027.

Khaspekov L, Friberg H, Halestrap A, Viktorov I, Wieloch T (1999) Cyclosporin $\mathrm{A}$ and its nonimmunosuppressive analogue N-Me-Val-4cyclosporin A mitigate glucose/oxygen deprivation-induced damage to rat cultured hippocampal neurons. Eur J Neurosci 11:3194-3198.

Kim JS, He L, Lemasters JJ (2003) Mitochondrial permeability transition: a common pathway to necrosis and apoptosis. Biochem Biophys Res Commun 304:463-470.

Kokoszka JE, Waymire KG, Levy SE, Sligh JE, Cai J, Jones DP, MacGregor GR, Wallace DC (2004) The ADP/ATP translocator is not essential for the mitochondrial permeability transition pore. Nature 427:461-465.

Kristal BS, Dubinsky JM (1997) Mitochondrial permeability transition in the central nervous system: induction by calcium cycling-dependent and -independent pathways. J Neurochem 69:524-538.

Kristian T, Gertsch J, Bates TE, Siesjo BK (2000) Characteristics of the calcium-triggered mitochondrial permeability transition in nonsynaptic brain mitochondria: effect of cyclosporin A and ubiquinone O. J Neurochem 74:1999-2009.

Matsumoto S, Isshiki A, Watanabe Y, Wieloch T (2002) Restricted clinical efficacy of cyclosporin A on rat transient middle cerebral artery occlusion. Life Sci 72:591-600.

McDonald JW, Goldberg MP, Gwag BJ, Chi SI, Choi DW (1996) Cyclosporine induces neuronal apoptosis and selective oligodendrocyte death in cortical cultures. Ann Neurol 40:750-758.

Mullen RJ, Buck CR, Smith AM (1992) NeuN, a neuronal specific nuclear protein in vertebrates. Development 116:201-211.

Nakagawa T, Shimizu S, Watanabe T, Yamaguchi O, Otsu K, Yamagata H, Inohara H, Kubo T, Tsujimoto Y (2005) Cyclophilin D-dependent mitochondrial permeability transition regulates some necrotic but not apoptotic cell death. Nature 434:652-658.

Nieminen AL, Petrie TG, Lemasters JJ, Selman WR (1996) Cyclosporin A delays mitochondrial depolarization induced by $N$-methyl-D-aspartate in cortical neurons: evidence of the mitochondrial permeability transition. Neuroscience 75:993-997.

Okonkwo DO, Melon DE, Pellicane AJ, Mutlu LK, Rubin DG, Stone JR, Helm GA (2003) Dose-response of cyclosporin A in attenuating traumatic axonal injury in rat. NeuroReport 14:463-466.

Pang Z, Bondada V, Sengoku T, Siman R, Geddes JW (2003) Calpain facilitates the neuron death induced by 3 -nitropropionic acid and contributes to the necrotic morphology. J Neuropathol Exp Neurol 62:633-643.

Schinder AF, Olson EC, Spitzer NC, Montal M (1996) Mitochondrial dysfunction is a primary event in glutamate neurotoxicity. J Neurosci 16:6125-6133.

Scorrano L, Ashiya M, Buttle K, Weiler S, Oakes SA, Mannella CA, Korsmeyer SJ (2002) A distinct pathway remodels mitochondrial cristae and mobilizes cytochrome c during apoptosis. Dev Cell 2:55-67.

Sengoku T, Bondada V, Hassane D, Dubal S, Geddes JW (2004) Tatcalpastatin fusion proteins transduce primary rat cortical neurons but do not inhibit cellular calpain activity. Exp Neurol 188:161-170. 
Sims NR (1990) Rapid isolation of metabolically active mitochondria from rat brain and subregions using Percoll density gradient centrifugation. J Neurochem 55:698-707.

Sinigaglia-Coimbra R, Cavalheiro EA, Coimbra C (2002) Protective effect of systemic treatment with cyclosporine A after global ischemia in rats. J Neurol Sci 203-204:273-276.

Sullivan PG, Rabchevsky AG, Waldmeier PC, Springer JE (2005) Mitochondrial permeability transition in CNS trauma: cause or effect of neuronal cell death? J Neurosci Res 79:231-239.

Szabo I, Zoratti M (1992) The mitochondrial megachannel is the permeability transition pore. J Bioenerg Biomembr 24:111-117.

Vachon P, Beaudry F, Marier JF, Ste-Marie L, Montgomery J (2002) Cyclo- sporin A in blood and brain tissue following intra-carotid injections in normal and stroke-induced rats. Brain Res 943:1-8.

White RJ, Reynolds IJ (1996) Mitochondrial depolarization in glutamatestimulated neurons: an early signal specific to excitotoxin exposure. J Neurosci 16:5688-5697.

Woodfield K, Ruck A, Brdiczka D, Halestrap AP (1998) Direct demonstration of a specific interaction between cyclophilin-D and the adenine nucleotide translocase confirms their role in the mitochondrial permeability transition. Biochem J 336:287-290.

Zoccarato F, Nicholls D (1982) The role of phosphate in the regulation of the independent calcium-efflux pathway of liver mitochondria. Eur J Biochem 127:333-338. 